\title{
Uma nova abordagem de um simulador virtual de identificação da qualidade da água baseado na metodologia de sistemas inteligentes inserido no contexto de redes neurais
}

\author{
Matheus S. Pestana* Orlando D. R. Filho** \\ Danúbia S. Pires ${ }^{* * *}$ \\ * Departamento de Eletroeletrônica, Instituto Federal de Educação, \\ Ciências e Tecnologia do Maranhão, MA, (e-mail: \\ pestana.m123@gmail.com). \\ ** Departamento de Eletroeletrônica, Instituto Federal de Educação, \\ Ciências e Tecnologia do Maranhão, MA, (e-mail: \\ orlando.rocha@ifma.edu.br) \\ *** Departamento de Eletroeletrônica, Instituto Federal de Educação, \\ Ciências e Tecnologia do Maranhão, MA, (e-mail: \\ danubiapires@ifma.edu.br)
}

\begin{abstract}
This paper is characterized as a nonlinear dynamic intelligent systems approach. It is proposed through the use of Artificial Neural Networks $(A N N)$ to a virtual system of analysis of the water potability standard. For the implementation of the proposal were used modeling and simulation software in order to identify and model a box type system. Using the learning and knowledge $A N N$ potencies to optimize the water treatment process. This strategy seeks to offer a system that assists in decision-making on the water quality index that is performed at Water Treatment Stations (WTA) ensuring reliability, robustness and efficiency about the final product that will be destined for distribution, avoiding errors and subjectivities. in the analyzes performed. In order to validate the developed methodology, the experimental results show that the proposal is very promising, presenting good computational performance and low response time.

Resumo: O presente artigo se caracteriza como uma abordagem de Sistemas Inteligentes dinâmicos de caráter não-lineares. Propõe-se através do uso de Redes Neurais Artificiais $(R N A)$ a um sistema virtual de análise do padrão de potabilidade da água, para a implementação da proposta foram utilizados softwares de modelagem e simulação com o intuito de identificar e modelar um sistema do tipo caixa preta, utilizando os potencias das $R N A$ de aprendizado e conhecimento para otimizar o processo de tratamento de água. Essa estratégia buscar oferecer um sistema auxiliar na tomada de decisão sobre o índice da qualidade da água que é realizado nas Estações de Tratamento de Água (ETA's) garantindo confiabilidade, robustez e eficiência acerca do produto final que será destinado à distribuição, evitando erros e subjetividades nas análises realizadas. Com o objetivo de realizar a validação da metodologia desenvolvida, os resultados experimentais demonstram que a proposta é bastante promissora, apresentando bom desempenho computacional e baixo tempo de resposta.
\end{abstract}

Keywords: Artificial Neural Networks, Water Treatment, Intelligent Systems, Computational Intelligence, Pattern Recognition

Palavras-chaves: Redes Neurais Artificiais; Tratamento de Água; Sistemas Inteligentes; Inteligência Computacional; Reconhecimento de Padrão. 


\section{INTRODUÇÃO}

A qualidade da água é definida como a resultante de fenômenos naturais e da atuação humana, tal interferência modifica suas características iniciais, tornando-a própria para a sua utilização em diversas atividades cotidianas Sperling (2017). A busca por processos de tratamento e análise de amostras cada vez mais puras, incentiva estudos de pesquisas para o desenvolvimento de novas tecnologias e procedimentos a fim de assegurar de forma dinâmica e robusta resultados mais confiáveis acerca da água que consumimos. O padrão de potabilidade de água possui destaque em diversos ramos da sociedade, sendo para o consumo diário, nas agroindústrias e nos processos industriais. Em diversas aplicabilidades, buscasse por recursos hídricos que possuem um padrão de pureza, com o objetivo de garantir a qualidade de produtos e serviços oferecidos. Necessita-se de ferramentas que possam avaliar a confiabilidade da água utilizada. A busca por uma metodologia eficiente, capaz de caracterizar a potabilidade da água após as etapas de realizadas nas Estações de Tratamento de Água - captação, coagulação, decantação, filtração, desinfecção e fluoretação - surge pelo alto grau de subjetividade e incerteza acerca do resultado final dos processos de purificação após as etapas de tratamento e distribuição para as áreas residenciais e industriais. O uso de uma plataforma de modelagem e simulação tem por objetivo realizar a interação homem e máquina de forma simples, auxiliando na tomada de decisão sobre o grau da qualidade da água após as etapas de tratamento baseados em valores préestabelecidos pelos órgãos de controle como o Ministério da Saúde e a Agencia Nacional de Águas. A análise de dados tem por objetivo prever um comportamento e de forma antecipada, informando ao operador para tomar a melhor decisão a fim reduzir o tempo de resposta, obter maior eficiência e garantir confiabilidade ao processo por inteiro. Nesse sentido, a metodologia proposta, oferece uma plataforma que busca utilizando os conceitos de Redes Neurais Artificiais $(R N A)$ avaliar de forma continua e eficaz para auxiliar o operador a tomar decisões com maior rapidez e segurança acerca da potabilidade da água, com uma modelagem que busca se aproximar ao máximo com o comportamento real.

As técnicas de Computação Inteligente, em especial as Redes Neurais Artificiais $(R N A)$, se mostram promissoras para processos que necessitam de técnicas de identificação e modelagem de sistemas dinâmicos podem ser descritas emBraga et al. (2007), Junior et al. (2007), Rezende (2003)

, Wang (1997), pois possuíam características como aprendizagem, treinamento, validação e teste (Haykin, 2001). As $R N A$ apresentam uma capacidade de reconhecimento de padrões, beneficiando processos de controle do tipo online, ou seja, quando o operador pode interferir de forma dinâmica Aguirre (2013), obedecendo os valores máximos permitidos pelos órgãos controladores.

\section{DEFINIÇÃO DA PROBLEMÁTICA}

O objetivo da Inteligência Artificial (IA) é o desenvolvimento de paradigmas ou algoritmos que requeiram máquinas para realizar tarefas cognitivas para as quais os humanos são altamente melhores Sage (1990). Uma IA deve haver três capacidades essenciais: armazenar conhecimento, aplicar o conhecimento armazenado e adquirir novos conhecimentos através de experiência. A IA, em especial Rede Neural Artificial, utiliza representação simbólica do conhecimento subjetivo, ou seja, de forma linguística regras, informações do especialista, especificações - para obter modelos lógicos que processam informação de forma qualitativa em computacional. Tais características de se mostram muito adequadas para a comunicação do tipo homem-máquina Coppin (2010).

Para a problemática analisada os conceitos de Inteligência Artificial se mostram como uma metodologia que possui a capacidade de promover de forma objetiva soluções dinâmicas para sistemas não lineares como podemos observar em Bond and Gasser (1998), Coppin (2010), Russel and Norvig (2013). Buscando novas técnicas com o objetivo de desenvolver novas ferramentas que auxiliem durante o processo de tratamento de água, reduzindo erros durante a tomada de decisões sobre a qualidade dos recursos hídricos a serem destinados aos consumidores, esta preocupação é evidenciada em cite Medina (1945), Chehade (2017), Yuanyuan Wang (2017).

O modelo proposto, possibilita transformar graus de subjetividade no padrão de potabilidade de água em informação quantitativa. Oferecendo a um sistema de análise e previsão baseados em Redes Neurais Artificias afim de auxiliar na caracterização do índice de qualidade de água otimizando o processo decisório e modelagem de sistemas do tipo não linear.

\section{FUNDAMENTAÇÃO TEÓRICA}

\subsection{Qualidade da Água}

A água é um dos recursos essenciais para a manutenção da vida no planeta. Através da água a sociedade moderna se desenvolveu como conhecemos hoje, auxiliando na fixação de grupos de pessoas próximas a rios e lagos. Atualmente buscasse cada vez mais por fontes hídricas para abastecer as cidades com altos graus de processos de tratamento dessa água pela poluição e contaminação nas zonas de captação em afluentes e rios. O Conselho Nacional do Meio Ambiente (CONAMA) através das resoluções 410 de 2009 e 430 de 2011, padrões de qualidade e parâmetros em limites aceitáveis de substancias com o objetivo de promover saúde as pessoas. O estudo busca garantir que os padrões sejam respeitados e auxiliando a avaliar a influência da interferência humana no resultado final do processo de captação, tratamento e distribuição pelas Estações de Tratamento de Água - ETAs. Fundamentado pela Índice de Qualidade de Águas do Ministério da Saúde ANA (2013), a água para ser considerada potável deve estar em conforme com o padrão microbiológico de padrões preestabelecidos de valor máximo permitido pelos órgãos regulamentadores competentes a fim de garantir a promoção, proteção e recuperação da saúde. Os parâmetros que são utilizados na elaboração deste estudo se baseia nos valores de Valores Máximos Permitidos $(V M P)$ que são estabelecidos pela Agencia Nacional de Águas em conjunto com o Ministério da Saúde, tais valores foram determinados via métodos de 
análise, com o intuito de oferecer aos consumidores um padrão de potabilidade da água destinada à população posterior ao processo de tratamento da água. Baseado nesses $V M P$, utilizamos os dados reais para realizar as simulações computacionais. Nesse sentido, a busca por uma ferramenta que seja uma alternativa para auxiliar na tomada de decisão acerca dos níveis de potabilidade, otimizando os processos de análise, podendo graduar o índice da qualidade da água capaz de evitar erros e subjetividade no serviço de tratamento nas ETAs.

\subsection{Redes Neurais Artificiais}

Segundo Haykin, uma rede neural é um processador maciçamente paralelamente distribuído constituído de unidades de processamento simples, que têm propensão natural para armazenar conhecimento experimental e torná-lo disponível para o uso. Elas possuem capacidades de organizar dados de forma estrutural de forma similar ao cérebro humano, de forma a realizar certos processamentos como reconhecimento de padrões, tais estruturas são conhecidas como neurônios. As estruturas de neuronais se assemelham ao cérebro humano nos aspectos de aprendizagem, uma vez que, o conhecimento é adquirido pela rede a partir do ambiente em que é inserido e na força de conexão entre os neurônios, denominadas como pesos sinápticos, onde o conhecimento adquirido é armazenado. O modelo não linear de um neurônio é matematicamente representado por (1) e (2) Haykin (2001).

$$
\begin{aligned}
& u_{k}=\sum_{k=1}^{m} w_{k j} x_{j} . \\
& y_{k}=\phi\left(u_{k}+b_{k}\right) .
\end{aligned}
$$

onde: $u_{k}$ é resposta para o k-ésimo neurônio; $m$ é o número de sinais de entrada do neurônio; $w_{k j}$ é o pesos sináptico associado ao k-ésimo neurônio; $x_{j}$ é o j-ésimo sinal de entrada do neurônio; $b_{k}$ é o bias ou limiar de cada neurônio; $y_{k}$ é o sinal de saída do k-ésimo neurônio; $\phi(k)$ é a função de ativação do k-ésimo neurônio.

O bias pode assumir valores positivos ou negativos, dependendo do sistema proposto, podemos modelar a saída alterando o bias. O seu uso tem o objetivo de aplicar uma transformação afim à saída $u_{k}$ e alterar o campo local induzido como demostrado em (3).

$$
v_{k}=\left(u_{k}+b_{k}\right) .
$$

onde $v_{k}$ representa o campo local induzido ou potencial de ativação para o neurônio k. O modelo neuronal pode ser expresso em blocos conforme a representação abaixo. Ver Figura 1.

Podemos identificar a partir do modelo neuronal, um conjunto de sinapses ou elos de conexão, individualmente qualificado pelo seu peso sináptico ou força própria, um elemento somador de sinais de entrada ponderados pelos pesos sinápticos do neurônio e uma função de ativação cuja função é restringir a amplitude da saída, tipicamente

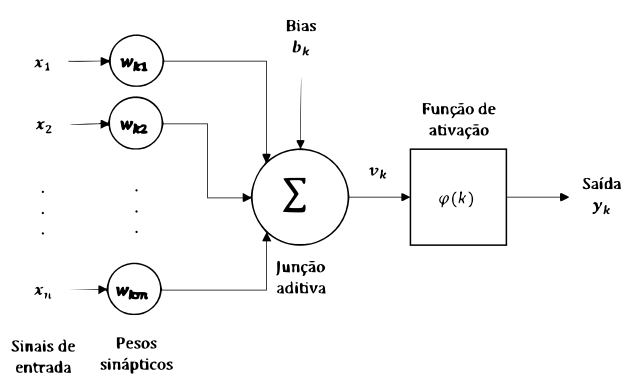

Figura 1. Modelo não-linear de um neurônio. Fonte: Autor. escrita em um intervalo unitário fechado [0, 1]. A propriedade fundamental de uma rede neural é a sua capacidade de aprendizagem, definida como o processo pelo qual os parâmetros livres de uma rede são adaptados através de um processo de estimulação pelo ambiente no qual está inserida. O tipo de aprendizagem é determinado pela maneira pela qual a modificação dos parâmetros ocorre Haykin (2001). Através da estimulação do ambiente, a rede é estimulada a realizar a modificações nos parâmetros e obter novas respostas aos sinais de entrada, sendo baseada na memória do processo.

As $R N A$ utilizam camadas de neurônios matemáticos que processam sinais de dados adquiridos do meio, essa informação é passada pelas demais camadas por meio de retro propagação e oferecendo uma saída de resposta a sua incitação inicial.

Quando trabalhamos com sistemas que utilizam as $R N A$, obtemos ferramenta poderosas capazes de receber, modelar e distribuir de maneira organizada diversas informações que são armazenadas e compartilhadas pela sua unidade de processamento. Durante o processo de aprendizagem, os pesos das conexões entre as respostas ao estimulo inicial são ajustados, proporcionando uma flexibilidade a modelagem de informação apresentada, distribuídas por meio da rede. A manipulação dessas informações por meio de interações nas unidades de processamento produzindo saídas.

O treinamento é supervisionado quando o ajuste dos parâmetros é feito com base na apresentação de um conjunto de pares de entrada e saídas padrão, nesse processo, uma entrada padrão é apresentada à rede e uma saída é calculada. O dimensionamento de uma rede neural depende de fatores como a quantidade de camadas e de neurônios que ela deve possuir, esses ajustes devem ser previamente definidos pelo especialista, uma vez que, o número de camadas e a quantidade de neurônios por camada é conhecido. As camadas intermediárias estão diretamente relacionadas a eficiência do treinamento, na qual o número de neurônios que a ela pertencem são determinantes para o seu funcionamento correto na solução de problemas. É proposto via o estudo, uma metodologia que relaciona as características de aprendizagem e treinamento das redes neurais para modelar um sistema capaz de reconhecer o padrão de potabilidade e auxiliar o operador a obter uma ferramenta confiável para tomada decisória acerca do resultado final do processo de tratamento de água.

\subsection{Identificação de Sistemas}

Identificação de Sistemas são definidos como uma área de modelagem matemática que estuda técnicas alternativas 
para descrever sistemas do tipo caixa branca, ou seja, quando é necessário o conhecimento das leis físicas que influenciam o seu funcionamento. No entanto, nem todos os sistemas analisados são conhecidos o seu comportamento conceitual pela física ou natureza, os estudos de modelagem e identificação quando se conhece o processo de atuação de forma parcial ou desconhece as características do sistema em questão, podemos analisa-lo com a abordagem do tipo caixa preta Aguirre (2013).

Para sistemas não lineares como as Redes Neurais Artificiais, a modelagem do tipo caixa preta é aplicada, pois os neurônios utilizados fazem a ponderação dos pesos sinápticos e o operador necessita apenas inserir os dados de entrada e saída do sistema, cabendo a ele determinar apenas a estrutura ou arquitetura de rede, o número de camadas, conexões necessárias e que dados irão ser utilizados no processo de aprendizagem e treinamento da $R N A$.

O estudo de identificação de sistemas decorre da ausência de informações previas do sistema de análise, seja por limitações de conhecimento, tempo, recursos para a estimação de parâmetros necessários para entendimento do conjunto de dados apurados. Nesse sentido, o uso das técnicas se mostraram essenciais para o desenvolvimento do presente projeto.

\section{METODOLOGIA}

O funcionamento da Rede Neural Artificial proposta para o estudo do padrão de potabilidade da água baseia-se no seguinte processo. A água em seu estado inicial é conduzida as Estações de Tratamento, nas quais os processos físicos e químicos são realizados como captação, coagulação ou floculação, decantação, filtração, desinfecção e fluoretação, após essa etapa, ou seja, na fase de análise final da água antes de ser destinada ao consumidor, onde são realizadas as verificações dos $V M P$ via analise químico-física, a metodologia proposta é inserida, com o objetivo de auxiliar na tomada de decisão acerca do padrão da qualidade da água analisada pelos laboratórios especializados ANA (2013). O estudo contempla o desenvolvimento de uma ferramenta auxiliar, que irá atuar após a fase de tratamento, uma vez que, o interesse dos autores é oferecer uma metodologia de análise, cujo intuito é buscar a uniformidade do resultado final de tratamento, com uma metodologia a ser aplicada no processo final dos processos realizados pelas ETAs, respeitando os VMP estabelecidos pela Agencia Nacional de Águas - ANA.

Por meio do Plano Nacional de Segurança Hídrica ( $P N S H)$ ANA (2019), a ANA por meio do Índice de Segurança Hídrica assegurar a analise integrada para solucionar os riscos de contaminação, preservação das propriedades originais e promover o respeito ao padrão de qualidade da água. A RNA irá graduar por meio de um Índice de Potabilidade se os valores de padrões microbiológicos estão de conforme com os predeterminados pelos órgãos de controle regulamentados por lei. Caso a amostra verificada estar em conformidade com o texto que regulamenta o padrão hídrico aceitável, ela poderá ser encaminha à distribuição, caso contrário, esta amostra será conduzida a fase de tratamento, com intuito de corrigir algum erro que ela possua. Ver Figura 2.

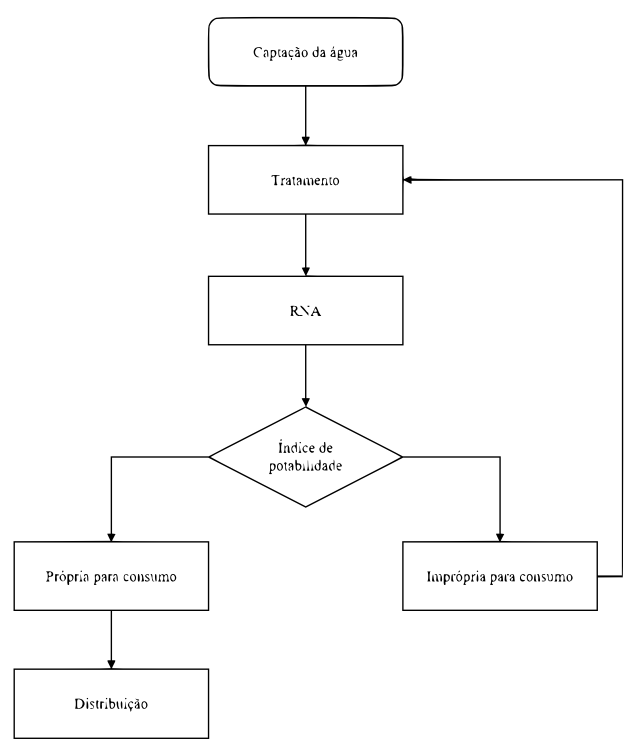

Figura 2. Fluxograma da metodologia proposta. Fonte: Autor.

\section{RESULTADOS COMPUTACIONAIS}

No desenvolvimento da Rede Neural Artificial foram utilizadas as arquiteturas de rede Cascade forward e Feedforward. Quando tratamos dados com valores muito próximos um do outro, o primeiro tipo de arquitetura de rede se mostra mais adequada, pois no seu processo de iteração, ela padroniza todas as entradas para uma matriz de dados de mesma ordem, proporcionando uma maior uniformidade nos dados e com isso oferendo uma medida de regressão linear melhor distribuída e facilitando o seu entendimento. A arquitetura do tipo Feedforward se torna mais aplicável após o tratamento inicial dos dados obtidos da rede do tipo Cascede forward, uma vez que, após a matriz e os pesos sinápticos estarem uniformizados, as iterações feitas pela Rede Neural se mostram mais eficientes para a análise, reduzindo erros e ruídos no processo de ponderação dos sinais de entrada e saída do sistema.

Utilizou-se os dados tabelados coletados da Agencia Nacional de Águas onde os parâmetros estão descritos e quantificados, esses dados foram modelados através de uma $R N A$ com arquitetura Cascade forward com duas camadas, sendo a primaria com 24 neurônios definidos pelo operador com 1000 ephocs durante o seu treinamento e validação dos dados e a segunda camada com dois neurônios, cujo o intuito é a validação dos dados da camada anterior. A $R N A$ apresentou uma característica de associação de padrão e oferecendo uma análise confiável para o estudo uma vez que a segunda camada é responsável pela caracterização da saída do sistema, podendo ser própria ou impropria ao consumo.

A metodologia se deu pela seguinte forma, os dados extraídos da Índice de Qualidade de Águas do Ministério da Saúde foram analisados criticamente, definido o seu comportamento e identificados os parâmetros decisórios para o funcionamento da rede. Os valores dos parâmetros são mostrados na Tabela 1.

Os valores dos sinais de entrada correspondem aos valores delimitados entre os valores máximos e mínimos. A saída 
do sistema corresponde à média das dos valores de entrada. Como mostrados na Tabela 2.

Embasado pelo Plano Nacional da Segurança Hídrica ANA (2019), com diretrizes que buscam por meio de estudos, planos e projetos assegurar reduzir os riscos de exposição e vulnerabilidade a contaminação por materiais nocivos ao consumo humano, causadores de doenças, como é o caso dos particulados abrangidos pela análise da turbidez. Para o consumo humano, o Potencial de Hidrogênio $(\mathrm{pH})$ assim como a temperatura dever ser normatizado, respeitando o padrão considerado próprio para sua utilização em seus diversos fins. A escolha por esses parâmetros de analise deu-se pela sua disponibilidade do mercado de encontrarmos sensores industriais capazes de realizar essas leituras com o intuito de realizar prototipagem.

Definidos as entradas e saídas, os vetores de pesos foram ajustados e obtido as seguintes regressões lineares, medidas que tomamos como variável para a análise das simulações obtidas, as figuras a seguir demonstram o comportamento entre as entradas e saídas, referidas com targets, a princípio analisadas de forma individualmente com o intuito de verificar o comportamento computacional das entradas e saídas. Ver Figuras 3, 4 e 5.
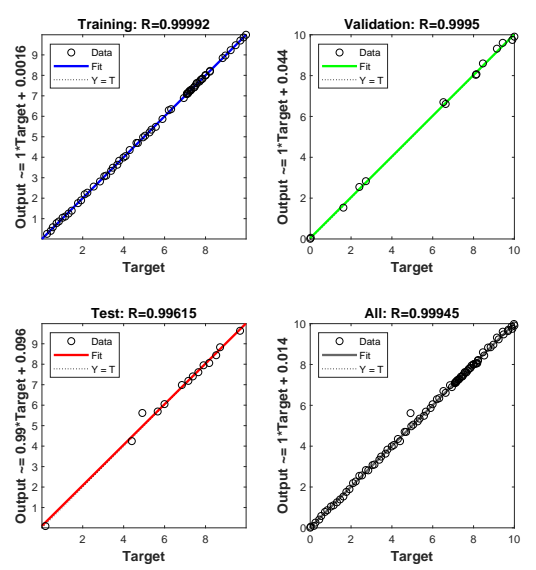

Figura 3. Regressão linear do parâmetro pH. Fonte: Autor.

Observa-se que no sistema estudado, para os dados colhidos pela Índice de Qualidade de Águas, os comportamentos dos parâmetros individuais se mostraram linearmente modelados e apresentando índices de performance consideráveis eficientes e confiáveis com índice de performance da rede de 0,88 utilizando 1000 iterações, representando

Tabela 1. Parâmetros de entrada da simulação. Fonte: ANA - Agencia Nacional de Águas.

\begin{tabular}{ccc} 
& Mínimo & Máximo \\
\hline Temperatura & $5^{\circ} \mathrm{C}$ & $30^{\circ} \mathrm{C}$ \\
Turbidez & OuT & $0,5 \mathrm{uT}$ \\
$\mathrm{pH}$ & 1 & 14 \\
\hline
\end{tabular}

Tabela 2. Parâmetros de saída da simulação. Fonte: Autor.

\begin{tabular}{ccc} 
& Mínimo & Máximo \\
\hline Temperatura & $20^{\circ} \mathrm{C}$ & $30^{\circ} \mathrm{C}$ \\
Turbidez & OuT & $0,15 \mathrm{uT}$ \\
$\mathrm{pH}$ & 5,9 & 7,5 \\
\hline
\end{tabular}
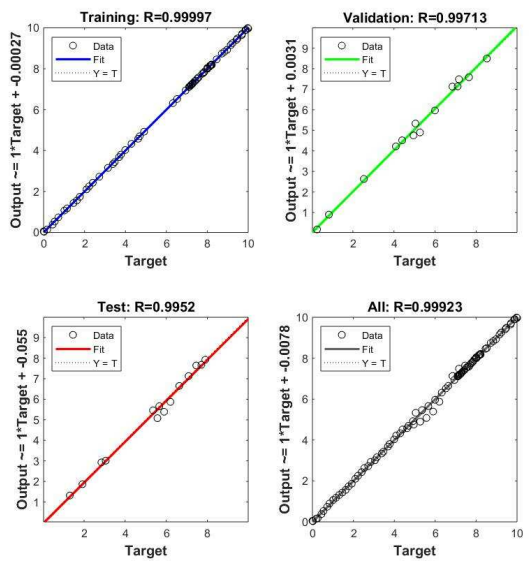

Figura 4. Regressão linear do parâmetro Temperatura. Fonte: Autor.
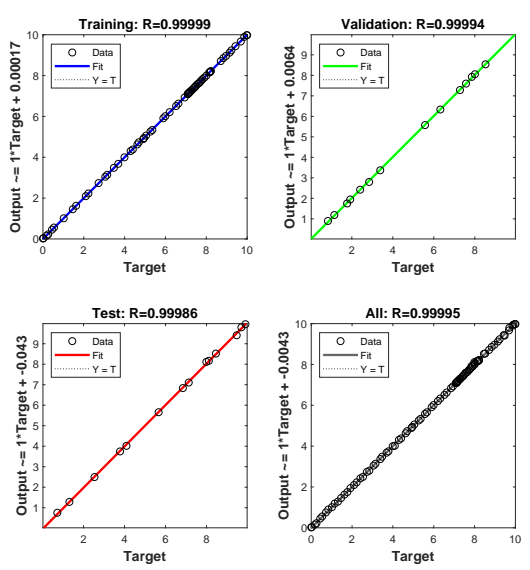

Figura 5. Regressão linear do parâmetro Turbidez. Fonte: Autor.

uma erro aceitável de aproximadamente 0,1 para sistemas de engenharia. Os valores da regressão linear relacionam os dados do vetor de entrada com os do vetor de target, a $R N A$ utiliza estes dados para a fazer as iterações matemáticas e oferecer uma saída ao estimulo de entrada. Quando os valores obtidos se aproximam de valores iguais a 1 , as regressões lineares que mais se aproximam, mostramse mais relevantes e melhores ponderados de acordo com a aprendizagem e treinamento da $R N A$. A arquitetura Cascade forward apresenta como vantagem a normatização dos vetores de entrada e de saída, que serão transformados em matrizes para então serem apresentados para a rede neural com todas as entradas e as saídas de igual ordem de elementos.

Após a verificação individual dos parâmetros, podemos avaliar seu comportamento em conjunto, observação suas características e particularidades, foi-se então desenvolvido uma $R N A$ com três entradas e duas saídas, ou seja, um sistema MIMO - multiples inputs and multiples outputs - que contém duas camadas de processamento neuronal, a primeira com 24 neurônios e a segunda com 2 neurônios. A primeira camada tem por objetivo realizar o aprendizado e treinamento, necessitando de um número maior de neurônios, a segunda, é encarregada de realizar a validação 
dos dados da camada anterior, necessitando de um número menor de neurônios para tal função. Podemos analisar o resultado da simulação realizada, ver Figura 6 .
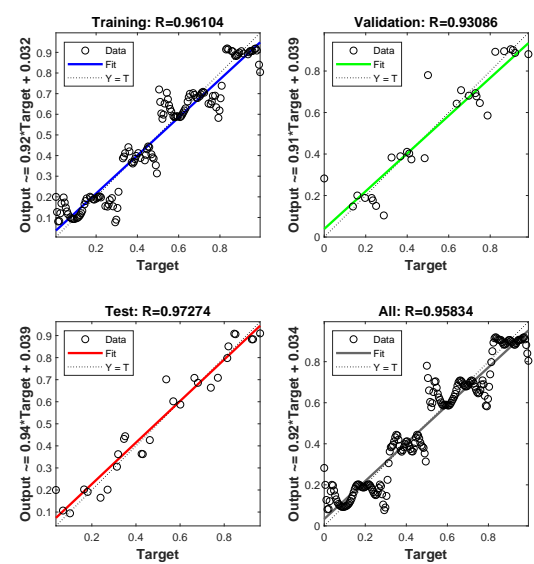

Figura 6. Rede neural com os parâmetros analisados em conjunto. Fonte: Autor.

A escolha das variáveis deu-se em recorrência de estudos acerca da disponibilidade de sensores e equipamentos a serem utilizados na indústria para sua implementação. Estes podem ser facilmente instalados e operados, garantindo confiabilidade aos processos de tratamento e analise da qualidade da água, os índices de performance e garantem a verossimilhança do comportamento das variáveis utilizados aplicado a modelagem caixa preta do sistema de analise virtual da qualidade de água após as etapas de tratamento.

Observamos que os resultados da regressão linear para a proposta se apresentam como uma metodologia eficiente, uma vez que, os dados obtidos na simulação demonstram a eficiência para a modelagem de sistemas do tipo não linear, oferecendo confiabilidade e robustez ao sistema de análise do tratamento da água.

\section{CONCLUSÃO}

O presente estudo se apresenta como uma metodologia que busca uma ferramenta que auxilie na tomada de decisão acerca da analise do padrão de potabilidade da água após o tratamento realizados nas Estações de Tratamento de Água. O uso da ferramenta computacional para a maior interação homem-máquina se configura uma mudança nos paradigmas de sistemas de análise e previsão para a área de controle.

Os dados obtidos via simulação virtual indicam a precisão e convergência dos sinais de entrada e resposta do sistema. Os algoritmos desenvolvidos se mostram eficazes no agrupamento de padrões durante os processos de aprendizagem, treinamento e validação. Os testes das simulações mostram a eficiência das Redes Neurais Artificiais para tratar dados de forma dinâmica, para sistemas do tipo on-line, quando o operador pode atuar diretamente na alteração de parâmetros. A abordagem utilizada se mostra bastante promissora para a problemática estudada para o reconhecimento de padrão da qualidade da água.

\section{AGRADECIMENTOS}

Os autores agradecem ao Programa Institucional de Bolsas de Iniciação Científica do Instituto Federal de Educação, Ciência e Tecnologia do Maranhão - Campus Monte Castelo e a Fundação de Amparo à Pesquisa do Estado do Maranhão (FAPEMA) pelo apoio financeiro e fomento para este presente estudo.

\section{REFERÊNCIAS}

Aguirre, L.A. (2013). Introdução à Identificação de Sistema: Técnicas Lineares e Não Lineares: Teoria e Aplicação. Editora UFMG, Brasil.

ANA (2013). Indicadores de Qualidade: Índice de Qualidade das Águas. Agencia Nacional de Águas - ANA, Brasil.

ANA (2019). Plano Nacional de Segurança Hídrica. Agencia Nacional de Águas - ANA, Brasil.

Bond, A.H. and Gasser, L. (1998). Reading in Distributed Artificial. Morgan Kaufmann Pub., EUA.

Braga, A.P., Carvalho, A.P.L.F., and Ludermir, T.B. (2007). Redes Neurais Artificiais: teoria e aplicações. Livros Técnicos e Científicos, Brasil.

Chehade, C.S..I.S..F.H. (2017). Smart technology for water quality control: Feedback about use of water quality sensors. 2017 Sensors Networks Smart and Emerging Technologies (SENSET), 35, 123-126.

Coppin, B. (2010). Inteligência artificial. Livros Técnicos e Científicos, Brasil.

Haykin, S. (2001). Redes Neurais: Princípios e prática. Bookman, Brasil.

Junior, H.A.O., Caldeira, A.M., Machado, M.A.S., Souza, R.C., and Tanscheit, R. (2007). Inteligência Computacional Aplicada à Administração, Economia e Engenharia em MATLAB. Thomson Learning Edições Ltda, Brasil.

Medina, A.D.D.V.E. (1945). Artificial intelligence model based on grey systems to assess water quality from santa river watershed. IEEE - Electronic Congress (E-CON $U N I)$.

Rezende, S.O. (2003). Sistemas Inteligentes: Fundamentos e Aplicações. Editora Manole, Brasil.

Russel, S. and Norvig, P. (2013). Inteligência Artificial. Elsevier Editora Ltda, Brasil.

Sage, A.P. (1990). Concise Encyclopedia of Information Processing in Systems ans Organization. Elsevier Science and Technology Books, Reino Unido.

Sperling, M. (2017). Identificação do estado trófico de lagos e reservatórios tropicais. Revista BIO, 3, 68-76.

Wang, L.X. (1997). A course in fuzzy systems and control. EUA.

Yuanyuan Wang, Jian Zhou, K.C.Y.W.L.L. (2017). Water quality prediction method based on lstm neural network. 12th International Conference on Intelligent Systems and Knowledge Engineering (ISKE). 